\title{
PULSED ION SOURCES OF DUOPLASMATRON TYPE WITH COLD AND HOT CATHODES
}

\author{
A. Glazov, V. Krasnopolsky, R. Meshcherov, V. Masalov, MRTI RAN
}

A description of $\mathrm{H}^{+}$ion sources construction and results of examination of their operating characteristics are presented. The beam energy is between 20 and $120 \mathrm{keV}$. The ion source with a hot cathode having a continuous bleeding of a working gas (the pressure in the discharge chamber is 0.02 Torr) operates at a pulse repetition frequency up to $25 \mathrm{~Hz}$ and a rectangular pulse length between 30 and $250 \mathrm{mcs}$. At the output of the source a ion beam $10 \mathrm{~mm}$ in diameter is formed. The amplitude of current pulse is up to $60 \mathrm{~mA}$. The ion energy is $60 \mathrm{keV}$ at a maximum pulse repetition frequency and a maximum pulse length. The source admits operating with a medium power consumption of $300 \mathrm{~W}$ during the discharge. The source is also used in a continuous regime at a lower beam current. The source with a cold cathode uses pulse gas bleeding in order to decrease gas consumption at a pressure $(0.2 \ldots 1)$ Torr in the discharge chamber. The main discharge current reaches the value between 100 and $170 \mathrm{~A}$ at a pulse length from 30 to $40 \mathrm{mks}$. The maximum output current equals from 0.8 up to $0.9 \mathrm{~A}$ at an extracting voltage of $90 \mathrm{kV}$.

\section{HOT - CATHODE ION SOURCE}

The source with a hot cathode of the type described in [ 1,2 ] is intended for use in installations with a small relative pulse duration. Therefore it uses the continious submission of working gas (hydrogen ) with the help of adjustable bleeding and the compulsory water cooling. For higher reliability in design the isolators made of ceramics and refractory metals are applied. The schematic diagram of the discharge chamber of the ion source and the accelerating gap is shown in Fig.1.

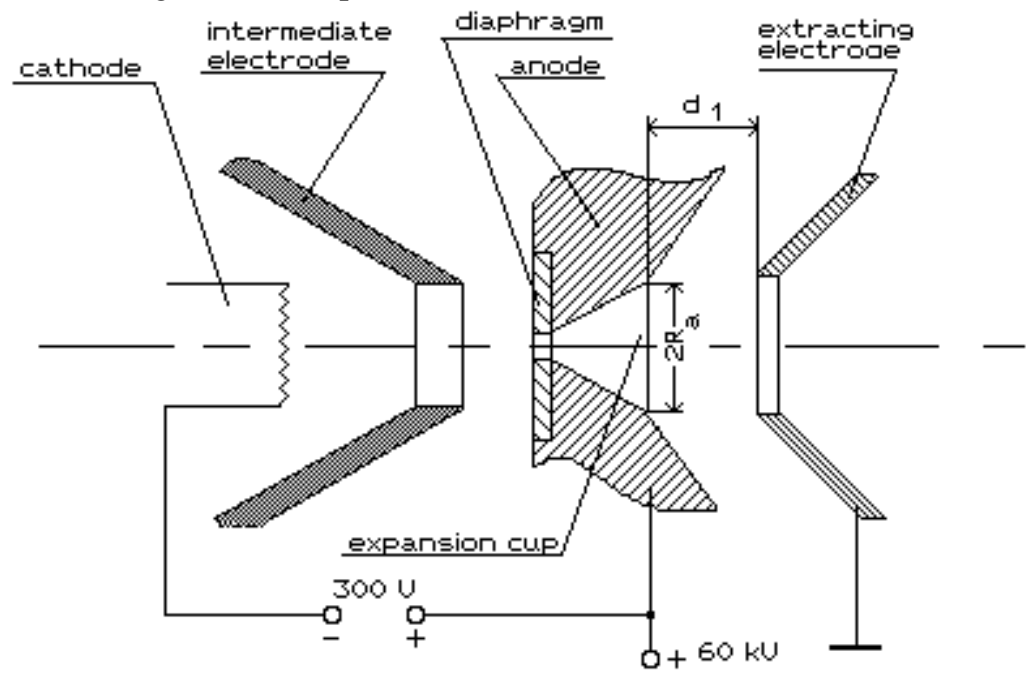

Figure 1. Schematic diagram of the discharge chamber of the ion source with hot cathode.

The working gas from the side of the hot cathode is supplied into the discharge chamber where the pressure of 0.02 Torr is supported as it is necessary for the charge existence.The pressure difference between the discharge chamber and the container with a pumping-out post ( $\mathrm{P}=1,5$ $10^{-5}$ Torr $)$ is reached by using a small hole ( $\left.1 \mathrm{~mm}\right)$ in the diaphragm. Within the gap between the intermediate anode and the main one the contracted magnetic field is created with the help of a magnetic coil. The design admits the disassembly into the main units: replacement of the hot-cathode, the diaphragm and the expansion cup. The ion source was tested in the frequency mode as well as in DC mode. At first the allowable level of the source power at which its long-term operation is possible was determined in the continious regime. It was found that at a constant power in the discharge up to $200 \mathrm{~W}(200 \mathrm{~V}, 1 \mathrm{~A})$ and the power supplied to the hot cathode and the magnetic coil of $200 \mathrm{~W}$ and $20 \mathrm{~W}$ respectively, the flow rate of cooling water of 1 litre/minute is enough. The constant voltage adjustable over the range ( $20 \ldots 60) \mathrm{kV}$ was applied to the accelerating gap of $13 \mathrm{~mm}$ wide. The frequency regime was provided by pulsed power supply of the discharge gap with rectangular pulses $250 \mathrm{mcs}$ in length and $300 \mathrm{~V}$ in amplitude. The pulse frequency as adjusted and reached $25 \mathrm{~Hz}$, at the same time the pulse amplitude of the discharge current did not exceed 12 A. The steady pulse 
height of the beam current of $65 \mathrm{~mA}$ at the lengh of $250 \mathrm{mcs}$ is achieved at the accelerating voltage of $60 \mathrm{kV}$, the average power of the discharge of $12 \mathrm{~W}$ and the aperture in the expansion cup of $9 \mathrm{~mm}$. The beam size at the distance of $200 \mathrm{~mm}$ from the source was not over $43 \mathrm{~mm}$ with no focusing elements. The experiments carried out with the help of collimating diaphragms and partitioned Faraday cup have allowed to evaluate the top level of the normalized emittance to be $\varepsilon=0,12 \pi \bullet \mathrm{cm} \bullet \operatorname{mrad}$. The source design allowed to carry out experiments in DC mode. The discharge start was executed with the help of a single voltage pulse as well as with a smooth change of a constant voltage on the intermediate electrode. At an extracting voltage up to $40 \mathrm{kV}$ the current of $\mathrm{H}^{+}, \mathrm{N}^{+}$ions was within the $\mathrm{mA}$-range. The experiments conducted permit to make the conclusion about the opportunity to use the presented source at high frequencies of current pulse repetition, therefore at a higher average current.

\section{COLD-CATHODE ION SOURCE}

The duoplasmatron with a cold cathode of the type described in [ 3 ] is schematically shown in Fig.2.

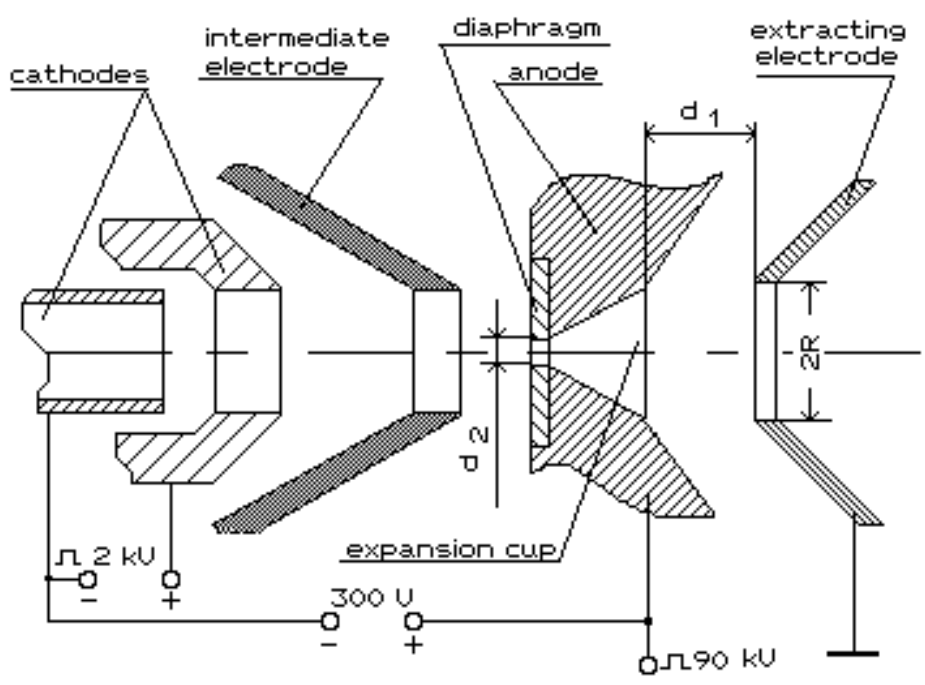

Figure 2. Schematic diagram of ion source with cold cathode.

It uses pulsed gas bleeding which is executed with the help of an electromagnetic valve. The construction of the valve permits to set up the regime of its operation during the experiment without the disassembly of the source. The opportunity of the pressure adjustment in the discharge chamber of the source within the interval $(0.2 \ldots 1.0)$ Torr is provided. The source can operate in a single pulse regime as well as in a frequency one as the system of water cooling is used. In comparison with the source considered above the hot cathode is replaced with a discharge cell using a cold hollow cathode made from molybdenum. The first discharge cell with a hollow cathode ( the cathode cell) is supplied with a voltage pulse up to $2 \mathrm{kV}$ and $10 \mathrm{mcs}$ long. The plasma which appears during the start plays the part of emitter for the next discharge cell ( the main) which is supplied with a voltage pulse up to $300 \mathrm{~V}$ and $100 \mathrm{mcs}$ long. The anode of the main cell has the emission aperture with the diameter from $1,3 \mathrm{~mm}$ to $2,6 \mathrm{~mm}$ which was changed during the experiments. The discharge in the main cell occures in the magnetic field which was changed up to $0,25 \mathrm{~T}$ during the experiment. The extracting voltage was of the pulsed type 90 $\mathrm{kV}$ in amplitude and $30 \mathrm{mcs}$ in length. The conducted investigations have allowed to determine the following optimum operating mode of the source: the current of the main discharge can vary from 140 to $150 \mathrm{~A}$; the delay time between the turn-on of the valve and the discharge equals
(4...5) ms. The optimum gap size between the intermediate electrode and the anode insert is equal to $5 \mathrm{~mm}$. At the source output the stable pulse amplitude of the current of $\mathrm{H}^{+}$beam was not less than $0.65 \mathrm{~A}$ at an extracting voltage of $90 \mathrm{kV} ; \mathrm{d}_{1}=25 \mathrm{~mm}, 2 \mathrm{R}=20 \mathrm{~mm}$ (see Fig.2). The diameter of the anode aperture is equa to $2,3 \mathrm{~mm}$. The control of the total current amplitude of $\mathrm{H}^{+}$beam was executed by the adjustment of the magnetic field as well as the value of the discharge current. The considered ion sources were tested in pilot operation and have confirmed their reliability and convenience in use.

\section{III.REFERENCES}

1. M.Ardenne "Tabellen der Elektronenphysik, Ionenphysik und Ubermikroskopic" Bd 1,2 Berlin, 1956.

2. I. V. Orfanov, V. A. Teplyakov "50 kV I on Tube" PTE (Pribory iTekhka Experimenta) 2, 150 (1960).

3. V. V. Nizhegorodtsev, Plasma I on Source, Author Certificate USSR No. 439232, published in Bulleten I zobreteney 27, 175 (1975). 